\title{
Efectos de los instrumentos de fomento exportador: algunas lecciones*
}

\author{
Roberto Álvarez Espinoza \\ Universidad de Chile
}

\section{Resumen}

Este trabajo ha tenido como objetivo extraer algunas lecciones sobre los efectos de la promoción de exportaciones en el desempeño de las firmas chilenas, utilizando varios estudios realizados durante la última década. En general, los distintos trabajos realizados, principalmente basados en la utilización de los instrumentos de PROCHILE, indican que la promoción de exportaciones ha tendido a favorecer el desempeño exportador de las firmas. Este resultado es relativamente robusto al uso de distintas fuentes de información y metodologías. Incluso, la utilización de técnicas modernas de evaluación de impacto revela efectos positivos de estos instrumentos. No obstante, esta literatura sugiere que los efectos tienden a ser heterogéneos por tipo de instrumento, por indicador de desempeño evaluado y por características de las empresas. Esto puede tener implicancias importantes para la implementación de este tipo de políticas.

Palabras clave: exportaciones, programas públicos, evaluación.

Clasificación JEL: F13, O19.

\section{Abstract}

The objective of this paper is to derive some lessons about the effects export promotion programs on the performance of Chilean firms, analyzing various studies carried out during the last decade. In general, these works, mainly based on the utilization of instruments managed by PROCHILE, indicate that promotion instruments have affected the firms export performance positively. These findings are robust to different information sources and methodologies. Moreover, recent findings using modern impact evaluation techniques show positive effects on this regard. The literature also suggests that these effects tend to be heterogeneous by type of instrument, by performance indicators and by firms characteristics. This evidence can have important implications for the design of these policies.

Keywords: exports, public programs, evaluation.

JEL classification: F13, O19.

\section{Introducción}

La mayoría de los países utilizan en algún grado instrumentos de fomento a las exportaciones. Existen varias justificaciones para la intervención del Estado en este ámbito, principalmente asociadas a ciertas fallas de mercado como externalidades en la información y en el uso de nuevas tecnologías en las firmas exportadoras. En

* Este trabajo es una versión extendida de la presentación «Permanencia exportadora y el impacto de los programas públicos», realizada por el autor en el IV Ciclo de Conferencias Cátedra de Economía Internacional, Universidad de Zaragoza, el 4 de mayo del 2011. El autor agradece la eficiente asistencia de investigación de Rolando Campusano. 
el caso de Chile, con la creación de la Agencia para la Promoción de Exportaciones (PROCHILE) han existido políticas explícitas de apoyo a las empresas exportadoras. El objetivo de esta agencia ha sido apoyar la política de diversificación y crecimiento de las exportaciones. Así como con otros instrumentos públicos, surgen interrogantes respecto a los efectos de estas políticas sobre el desempeño de las firmas y en qué ámbitos se han manifestado. Por ello, el objetivo de este trabajo es hacer un resumen de la literatura en el caso de Chile, discutiendo los resultados de varias evaluaciones que se han realizado y las implicaciones de política que surgen de esto estudios.

La evaluación de estas políticas y sus instrumentos enfrenta varias dificultades. En primer lugar, no es trivial identificar cuáles serían los efectos esperados. En general, la mayoría de los instrumentos se establecen con objetivos más o menos generales que dificultan su evaluación. En segundo lugar, los investigadores se encuentran con problemas asociados con los datos necesarios para la realización de estas evaluaciones. En el mejor de los casos, las agencias cuentan con información de los beneficiarios de estos instrumentos, pero no cuenta con información para una muestra de empresas que pueda actuar como un grupo de control. Además, aun cuando se pueda contar con este tipo de datos, comúnmente la disponibilidad de información sobre características de las firmas es relativamente limitada. Finalmente, dada estas limitaciones, la evaluación de impacto presenta desafíos metodológicos no menores. Debido a ello, las evaluaciones formales de los efectos de instrumentos de promoción de exportaciones son más bien escasas y con muestras pequeñas de empresas.

Existen varios estudios en países desarrollados, como los de Wilkinson y Brouthers (2000), Cavusgil y Naor (1987), y Coughlin y Cartwirght (1987), que sugieren una relación positiva entre desempeño exportador y los programas públicos en Estados Unidos. En el caso de instrumentos específicos, Spence (2003) analiza el impacto de las misiones comerciales en el Reino Unido y sugiere que éstas han contribuido positivamente al incremento de las exportaciones de las firmas participantes. El impacto de la participación en ferias de muestras ha sido analizado por Blythe (1996), Pfeiffer, Burgemeister, Hibbert y Spence (1998), Shipley, Egan y Wong (1993) y más recientemente por Wilkinson y Broughters (2006).

Para países en desarrollo, a pesar de la importancia de este tema, la evidencia ha sido más escasa, destacándose recientemente los trabajos realizados por el Banco Interamericano del Desarrollo, que estudian los efectos de los instrumentos de fomento exportador administrados por agencias públicas en seis países latinoamericanos (Perú, Costa Rica, Chile, Colombia, Argentina y Uruguay) y utilizando metodologías formales de evaluación de impacto (Volpe Martincus, 2010) ${ }^{1}$.

1 Entre las contribuciones previas, pero usando datos agregados, destacan los trabajos de ARSLAN y VAN WIJNBERGEN (1993) y LOW (1982). 
El trabajo es estructurado de la siguiente manera. En la segunda sección se presenta una discusión de los fundamentos teóricos para la adopción de políticas de promoción de exportaciones. La tercera sección resume la evidencia empírica en Chile acerca de los efectos de estos instrumentos. La cuarta sección concluye.

\section{Justificación de las políticas de promoción de exportaciones}

Los instrumentos de apoyo a las exportaciones se han justificado por la existencia de ciertas fallas en los mercados, tales como las externalidades de información y tecnológicas que serían generadas por las exportaciones (Lederman et al., 2010). En el caso de la primera, dado que no existe perfecta información respecto a las preferencias de los consumidores, las oportunidad de negocios, las calidad y requerimientos técnicos para exportar en los mercados externos, los que primero exportan a un mercado en particular revelan información relevante que no es completamente apropiada por el pionero (Hausmanny Rodrik, 2003; Westphal, 1990). Por ello, existiría poca inversión ex-ante en penetrar los mercados internacionales y mucha entrada ex-post, lo que reduce los niveles de inversión por debajo de su óptimo social.

En el caso de la externalidades tecnológicas, el argumento es que los exportadores pueden reducir los costos de producción a otras firmas a través de la transferencia no intencional de nuevas tecnologías y/o productos hacia otras firmas. Este argumento es similar al de las externalidades en la literatura de innovación e I+D, y ocurre cuando la inversión en nuevas tecnologías, que en este caso sería más relevante en el caso de firmas que venden en los mercados internacionales, tampoco es completamente apropiada por el innovador. En el caso de los países en desarrollo incluso podría argumentarse que este mecanismo sería más importante por cuanto las ventas al exterior, especialmente a países más desarrollados, incentivarían la adopción de nuevas tecnologías y el desarrollo de nuevos productos para clientes más exigentes que los del mercado interno.

Varios autores han presentado argumentos respecto a que los exportadores tienden a adoptar estilos más eficientes y competitivos de gestión, y realizan una mayor capacitación de sus trabajadores, lo que puede beneficiar a otras empresas (Keesing, 1967; Feder, 1982; Edwards, 1993 ${ }^{2}$. La existencia de estas externalidades y su justificación para la promoción de exportaciones es, sin embargo, controvertida. Los partidarios de políticas activas de promoción de exportación han utilizado tales justificaciones para apoyar la intervención del gobierno. Lall (2002), por ejemplo, indi-

${ }^{2}$ La mayor parte de estos argumentos no han sido formalizados en modelos teóricos. Esto contrasta con modelos que consideran a las empresas multinacionales como fuente de externalidades para las empresas domésticas. Véanse, por ejemplo, RODRIGUEZ-CLARE (1996), MARKUSEN y VENABLES (1999) y MARKUSEN y TROFIMENKO (2007). 
ca que la evidencia empírica sugiere que estas políticas han sido efectivas para mejorar el desempeño exportador de las economías de industrialización reciente. No obstante, los escépticos argumentan que estas políticas pueden tener efectos distorsionadores sobre la competencia y atentan contra el sistema multilateral de libre comercio. Panagariya (2000), por ejemplo, discute que los argumentos tradicionales y recientes no son convincentes desde un punto de vista teórico y empírico para implementar políticas selectivas para la promoción de las exportaciones, mientras Rodrik (1999) señala que no hay evidencia robusta de externalidades provenientes de las exportaciones.

Esta controversia implica que la evidencia empírica es crucial para dilucidar si estas políticas están justificadas o no. Existe una literatura relativamente abundante que analiza si la mayor presencia de exportadores en una región y/o o sector productivo tiene algún efecto sobre el desempeño exportador y/o la productividad de otras firmas. En la mayoría de estos trabajos, se distingue entre la actividad exportadora de empresas domésticas y multinacionales, y se analiza si la mayor concentración geográfica y/o sectorial de estos exportadores afecta variables relativas al desempeño exportador (probabilidad de exportar y exportaciones sobre ventas) de las firmas en el mismo sector o sectores relacionados. En este último caso, a partir del trabajo de Javorcik (2004), se distingue entre externalidades horizontales (misma industria) y verticales (industrias que demandan o proveen insumos).

En el Cuadro 1, presentado por Álvarez y López (2008), se muestra un resumen de algunos estudios que han evaluado la presencia de externalidades asociadas a la actividad exportadora. La evidencia empírica sobre la existencia de estas externalidades es mixta (Greenaway y Kneller, 2007). Existen algunos estudios que no encuentran que la actividad exportadora en un sector afecta el desempeño de las firmas (Clerides et al., 1998; Barrios et al., 2003; Bernard y Jensen, 2004). Otros, en cambio, han encontrado evidencia de un efecto positivo sólo de la actividad exportadora de empresas multinacionales (Aitken et al., 1997; Greenaway et al., 2004; Ruane y Sutherland; 2005).

En el caso de Chile, Álvarez y López (2008) analizan el efecto de la actividad exportadora en la misma industria e industrias relacionadas, utilizando información de establecimientos manufactureros en el período 1990-1999. Se encuentra evidencia que las exportaciones de firmas domésticas y extranjeras en una industria aumentan la productividad de firmas en las industrias que le proveen insumos. Sus resultados sugieren la existencia de externalidades tecnológicas dentro de una misma industria (horizontales), pero sólo para el caso de las exportaciones de empresas de propiedad extranjera.

Esta evidencia no concluyente respecto a la existencia de externalidades puede ser explicada no sólo por las distintas metodologías aplicadas, sino también por la variedad de indicadores que han sido utilizados para medir la intensidad exportadora (Greenaway y Kneller, 2007). No obstante lo anterior, también existen dificultades metodológicas para identificar econométricamente el efecto causal de la actividad exportadora regional y/o sectorial sobre el desempeño de otras firmas. 
CUADRO 1

RESUMEN DE ESTUDIOS SOBRE EXTERNALIDADES DEL SECTOR EXPORTADOR

\begin{tabular}{|c|c|c|c|}
\hline & $\begin{array}{c}\text { Probabilidad de exportar } \\
\text { y/o } \\
\text { Intensidad exportadora } \\
\text { (exportaciones/ventas) }\end{array}$ & $\begin{array}{c}\text { Pxportadores } \\
\text { extranjeros }\end{array}$ & $\begin{array}{c}\text { Exportadores } \\
\text { nacionales }\end{array}$ \\
\cline { 3 - 4 } $\begin{array}{c}\text { Horizontal } \\
\text { (misma industria) }\end{array}$ & $\begin{array}{c}\text { AHH, CLT, BGS, } \\
\text { GK, S, GSW, BJ, } \\
\text { RS, KK, KP }\end{array}$ & CLT, GGP, GH & CLT \\
\hline $\begin{array}{c}\text { Backward } \\
\text { (compra insumos) }\end{array}$ & KP & J, GGP & \\
\hline $\begin{array}{c}\text { Forward } \\
\text { (vende insumos) }\end{array}$ & KP & - & \\
\hline
\end{tabular}

FUENTE: Álvarez y López (2008). AHH: Aitken, Hanson and Harrison (1997); CLT: Clerides, Lach and Tybout (1998); BGS: Barrios, Görg and Strobl (2003); GK: Greenaway and Kneller (2008); S: Sjöholmm (2003); GSW: Greenaway, Sousa and Wakelin (2004); BJ: Bernard and Jensen (2004); RS: Ruane and Sutherland (2005); KK: Karpaty and Kneller (2005); KP: Kneller and Pisu (2007); GGP: Girma, Görg and Pisu (2008); GH: Görg and Hijzen (2004); J: Javorcik (2004).

\section{Análisis de los estudios realizados en Chile}

En esta sección se analizan los principales estudios que se han realizado para evaluar el impacto de instrumentos de promoción de exportaciones en el caso de Chile. Cabe destacar que este tipo de evaluaciones enfrentan varias dificultades metodológicas que es necesario tener presente al analizar sus resultados. Primero, es necesario definir los efectos esperados de un programa al evaluarlo. En general, la mayoría de los instrumentos se establecen con objetivos más o menos generales que dificultan su evaluación. Segundo, existen problemas asociados a la obtención de información necesaria para la realización de estas evaluaciones. En el mejor de los casos, las agencias cuentan con información de los beneficiarios de estos instrumentos, pero no cuentan con información para una muestra de empresas que pueda actuar como un grupo de control. Además, aun cuando se pueda contar con este tipo de datos, comúnmente la disponibilidad de información sobre características de las firmas es relativamente limitada. Finalmente, la necesidad de recabar este tipo de información requiere realizar encuestas a las empresas cuyo elevado coste determina que la evaluación se realice con una muestra reducida de empresas. 
Los primeros estudios realizados en Chile evaluaron los instrumentos de fomento exportador de PROCHILE $^{3}$, que han estado orientados hacia la diversificación de los productos exportados, los mercados de destino y las empresas exportadoras, el incremento de los volúmenes exportados y el acceso a posiciones cada vez más competitivas en la cadena de comercialización. De acuerdo a sus objetivos, esta agencia organizaba sus programas en función de tres áreas principales: (i) Campaña de Posicionamiento Económico (CPE): promoción de la imagen del país en mercados externos, (ii) Programa de Promoción de Exportaciones (PPE) y (iii) Sistema de Información Comercial (SIC)

La CPE tenía como objetivo de difundir, fortalecer y posicionar la imagen del país en los mercados externos. Estas campañas implican promocionar la «marca Chile», a través de medios masivos de comunicación en los mercados objetivos. En esta actividad, dada su naturaleza, no participaban en forma individual empresas beneficiarias. La CPE se ha estructurado en base a la participación de la Institución conjuntamente con otras entidades como las asociaciones gremiales, que cofinancian el costo de cada campaña.

El objetivo del PPE era obtener resultados concretos en materia de desempeño exportador de las empresas, fundamentalmente a través de actividades de penetración y diversificación de mercados. Las actividades enmarcadas en el PPE, también se realizaban en conjunto con el sector privado, el que ha participado activamente a través del cofinanciamiento de las mismas o en la operación del diseño e implementación de campañas de promoción internacional. La modalidad habitual de trabajo, aunque de manera no exclusiva, se relaciona con programas de promoción y comercialización implementados a través de los comités de exportadores.

Los comités son agrupaciones de empresarios con objetivos comunes en el ámbito de negocios internacionales. Éstos están constituidos al menos por cuatro empresas y nacen a partir de las peticiones de asociaciones gremialeso, en su defecto, como resultado de los esfuerzos de promoción de PROCHILE, institución que apoya la asociatividad de empresarios de un mismo sector. Las principales actividades realizadas en el marco de los comités exportadores han sido las misiones comerciales, misiones de prospección de mercados, edición de material gráfico promocional (folletos, videos, catálogos, etc.), participación y visitas a ferias, participación y asistencias a eventos internacionales, estudios de mercado e invitación a compradores, autoridades y expertos. Sin embargo, en el marco de los PPE también se ha organizado la asistencia a ferias internacionales por parte de empresas que no necesariamente pertenecen a un comité exportador, aunque sí están integradas a asociaciones gremiales con las que PROCHILE ha coordinado la asistencia a las ferias para la promoción de sus productos y servicios.

${ }^{3}$ PROCHILE es un organismo público cuyo objetivo es apoyar el proceso de internacionalización de la economía chilena, a través del fomento de las exportaciones no tradicionales, tanto de bienes como de servicios. 
Finalmente, el único ámbito donde, en forma estilizada, puede admitirse una cierta participación individual es en relación a aquellas empresas interesadas en la obtención de información específica de los mercados internacionales. Esta información se ha canalizado a través del Sistema de Información Comercial (SIC). Generalmente tiene que ver con información referida a precios, costos de transportes, normas de ingreso y aranceles en los mercados internacionales.

Con el paso de los años, PROCHILE ha ido modificando su estructura y los instrumentos de promoción de exportaciones. Actualmente, lo hace principalmente a través de los siguientes instrumentos:

1. Estudios a pedido: estudios realizados a partir de las diversas fuentes de información de PROCHILE, cada empresa recibe una atención personalizada de acuerdo a sus necesidades específicas de información.

2. Participación en ferias internacionales: esta herramienta le permite a las empresas ser expositores en los principales eventos de su rubro, amparado y respaldado en un Pabellón País. Entre los servicios que contempla se considera el soporte técnico en los mercados de destino, organización del stand y su presentación, reserva de espacios, desarrollo de merchandising, traductores especializados y coordinación de reuniones con potenciales importadores.

3. Concurso de promoción de exportaciones: el objetivo de este concurso es apoyar estrategias de promoción de exportaciones de los sectores silvoagropecuario y de productos del mar. Se contempla cofinanciamiento público de ambos sectores.

4. Curso Chile Branding: cuyo objetivo es poner a disposición de las empresas nacionales las últimas tendencias de posicionamiento de marca utilizadas en los mercados más influyentes del mundo.

5. Diploma e-learningen comercio internacional: capacitación para las pequeñas y medianas empresas en materias de comercio internacional mediante actividades interactivas y colaborativas que permitan la adquisición de aprendizajes significativos y permanentes que apoyen el desempeño de los empresarios nacionales en el contexto del comercio exterior.

6. Talleres de Formación: contemplan actividades como la realización de talleres, charlas técnicas, traída de expertos, publicaciones de interés para un sector, y actividades de inteligencia de mercado, entre otros. Estos talleres son sin costo para los participantes.

7. Programa de pre-internacionalización: herramienta de aprendizaje en marketing, comunicación, relaciones públicas y ventas. Las postulaciones y costo varían según la feria en la que le interese participar.

8. Coaching exportador: enfocado en potenciar las habilidades de liderazgo y gestión de nuevos negocios de los participantes, y apoyarlos en la construcción de redes de apoyo para fortalecer su capacidad exportadora.

9. Diplomado en comercio internacional: capacitación que facilita la formación, aprendizaje, preparación y mejoramiento de la calidad de las empresas en la 
gestión internacional, con la finalidad de incorporar y desarrollar nuevos exportadores de vanguardia.

Los primeros trabajos realizados en Chile evaluando estos instrumentos se concentraron en las actividades contempladas en el PPE y la utilización del sistema de información comercial, sin indagar mayormente en otras actividades como la CPE que no contemplaba la participación específica de empresas. Alvarez y Crespi (2000a)utilizan información de 365 empresas exportadoras, que fueron seleccionadas del universo de empresas con participación en los mercados internacionales, y separadas en una muestra de tratamiento y control ${ }^{4}$. Una encuesta a las empresas de ambos grupos fue realizada para indagar en información cualitativa no disponible, cómo los esfuerzos en innovación y prácticas en negocios internacionales, y en información cuantitativa como empleo y ventas que no se encuentra habitualmente disponible en los registros de exportación.

Sus resultados indican que la utilización de los instrumentos de promoción genera un impacto positivo y significativo en el número de mercados de exportación. De esta evidencia se puede inferir que los instrumentos de PROCHILE han permitido un incremento de aproximadamente un mercado en promedio para las firmas participantes, en comparación con el grupo de control. En relación con las otras variables de desempeño analizadas; introducción de nuevos productos y crecimiento de las exportaciones, no se encuentra un impacto estadísticamente significativo. Sin embargo, sus resultados indican que la utilización de estos instrumentos genera un impacto positivo indirecto sobre el crecimiento de las exportaciones, ya que el cambio en el valor exportado es afectado positivamente por el número de mercados al inicio. En efecto, si en promedio los instrumentos de PROCHILE permiten exportar a un mercado adicional y, si además, como se desprende de las estimaciones, un mercado adicional incrementa las exportaciones en aproximadamente 200 mil dólares, se puede inferir que, en un período de cuatro años, estos instrumentos generan tal aumento en las exportaciones de las firmas que los utilizan.

El estudio realiza además un análisis comparativo del impacto diferenciado de los tipos de instrumentos. Por ello, se comparan los resultados de las empresas que han participado en comités de exportación con el resto de las empresas, que sólo han usado los otros instrumentos (ferias y sistemas de información comercial). Los resultados de estas estimaciones, permiten inferir que, independiente del tipo de instrumentos utilizados, la participación en PROCHILE tiene un impacto positivo sobre el número de mercados, no así sobre el resto de los indicadores de desempeño. No obstante, el impacto de la participación en los comités es mayor que el de la utilización de los otros tipos de instrumentos. En efecto, los comités permiten un incremento promedio de aproximadamente 1,5 mercados. En cambio, las ferias y el

${ }^{4}$ La información base corresponde a variables de exportaciones (valor exportado, numero de productos exportador y mercados de destino) y el tamaño de las muestra de tratamiento y control son 178 y 187 , respectivamente. 
sistema de información comercial incrementarían casi en uno el número de mercados de exportación ${ }^{5}$.

En Álvarez y Crespi (2000b) se utiliza la misma fuente de información, y se indaga sobre los factores de carácter cualitativo (innovación y gestión internacional) que pueden ser afectados por la utilización de instrumentos de fomento exportador. Sus resultados sugieren que la utilización de estos instrumentos ha estado positivamente asociada a una mayor innovación de las empresas. De hecho, comparado al grupo de control, las empresas beneficiarias declaran haber innovado más en términos de mejoramiento tecnológico de los productos, introducción de nuevos productos y cambios organizacionales ${ }^{6}$. También se encuentra que estos instrumentos se asocian positivamente con mayores esfuerzos internos en el mejoramiento de la capacidad exportadora de las empresas, como por ejemplo, en el establecimiento de alianzas estratégicas con clientes y proveedores, contratación de personal cualificado y capacitación del personal, mayor inversión en promoción externa y mejoramientos en los sistemas de información comercial.

En ambos casos, la metodología de evaluación es similar. Primero, se estima un modelo Probit para determinar la probabilidad de haber utilizado los instrumentos de promoción, y luego se selecciona una muestra de control que sea lo más parecida posible al grupo de beneficiarios. Segundo, se estima el efecto del tratamiento mediante una estimación de corte transversal para las variables de desempeño (exportaciones, innovación, etc.) medidas con posterioridad al tratamiento. Dado ello, estos trabajos sugieren que existe alguna evidencia de una relación positiva entre los instrumentos y el desempeño exportador e innovador de las empresas, pero no son suficientemente concluyentes sobre las relaciones de causalidad.

El estudio de Álvarez (2004) es similar a los anteriores, pero con una motivación distinta. Al igual que los trabajos previos, se construye una muestra de empresas tratadas y una muestra de control. Suponiendo que las principales diferencias en la probabilidad de usar estos instrumentos están dadas por factores observables, estas características se incluyen como variables de control en la ecuación de resultado ${ }^{7}$. No obstante, se diferencia en dos aspectos fundamentales de los estudios anteriores. Primero, su énfasis está en los efectos de los instrumentos de promoción sobre el comportamiento exportador de las PYMES. Segundo, es motivado por el hecho que este segmento de empresas tiene una probabilidad menor de ser un exportador permanente. Como se puede apreciar en el Cuadro 2, la probabilidad de exportar es menor en las pymes que en las empresas grandes: sólo un 14 por 100 de las empre-

5 Más aún, el impacto de estos dos últimos instrumentos deja de ser significativo en algunas especificaciones, lo que no ocurre con el parámetro asociado a la participación en comités.

${ }^{6}$ No se encuentran efectos sobre innovación de procesos. Esto sugiere que las empresas exportadoras intentando mejorar su posicionamiento internacional parecen más orientadas hacia el mejoramiento de sus productos que a sus procesos productivos.

7 Este es un supuesto bastante fuerte dado que la selección puede estar dada por factores no observados. La metodología de diferencias en diferencias aplicada recientemente en el caso Chileno, y que se discute más abajo, son más adecuadas para solucionar este problema. 
sas pequeñas había exportado en el período 1990-1996. En cambio, más del 74 por 100 de las empresas grandes exportó en el mismo período. Más aún, las pymes son menos capaces de consolidarse como exportadores permanentes. El porcentaje de exportadores permanentes en el segmento de pequeñas y medianas empresas alcanza al 1 por 100 y 11 por 100, respectivamente. En contraste, en las empresas de gran tamaño, los exportadores permanentes representan un 30 por 100 de las empresas en este grupo.

\section{CUADRO 2}

DESEMPEÑO EXPORTADOR Y TAMAÑO DE EMPRESAS

\begin{tabular}{|l|c|c|c|c|c|r|}
\hline \multirow{2}{*}{\multicolumn{1}{c|}{ Estatus exportador }} & \multicolumn{2}{c|}{ Pequeña } & \multicolumn{2}{c|}{ Mediana } & \multicolumn{2}{c|}{ Grande } \\
\cline { 2 - 7 } & N & \% & N & $\%$ & \multicolumn{1}{c|}{ N } & $\%$ \\
\hline No exportador & 4.284 & 86 & 780 & 48 & 132 & 26 \\
Exportador esporádico & 650 & 13 & 659 & 41 & 220 & 43 \\
Exportador permanente & 47 & 1 & 185 & 11 & 164 & 32 \\
Total & 4.981 & 100 & 1.624 & 100 & 516 & 100 \\
Esporádico/Total exportadores & - & 93 & - & 78 & - & 57 \\
\hline
\end{tabular}

FUENTE: Álvarez (2004), basado en la Encuesta Anual de Manufacturas Los no-exportadores se definen como aquellas empresas que no exportaron durante ningún año del período 1990-1996, exportadores esporádicos son aquellos que exportaron en algún año de este período y permanentes exportadores son ésos que exportaron en todos los años de este período.

Basado en este evidencia, Álvarez (2004) analiza los factores que explican la persistencia de la firmas pequeñas y medianas en los mercados de exportación, si la participación en programas de fomento exportador aumenta la probabilidad de permanecer exportando y si existen diferencias en el efecto de los distintos programas. Para determinar la muestra de empresas a estudiar, se selecciona a todos los exportadores pequeños (10-50 trabajadores) y medianos (51-20 trabajadores) en la industria manufacturera Chilena durante el período 1990-1996 y se define dos tipos de exportadores: permanentes (exportaron todos los años del período) y esporádicos (exportaron algún año en ese período) ${ }^{8}$.

Los resultados de las estimaciones indican que la probabilidad de ser un exportador permanente no se ve afectada por la utilización de instrumentos de PROCHILE, pero sí por la utilización de otros instrumentos de fomento exportador ${ }^{9}$. Ade-

8 La información utilizada en las estimaciones proviene de una encuesta especial aplicada a una muestra aleatoria de 295 empresas, elegida de a acuerdo a estratificación sectorial y margen de error del 5 por 100, correspondiente a 157 exportadores permanentes y 138 exportadores esporádicos.

9 Existen otros instrumento de fomento exportador no administrados por PROCHILE como los reintegros a los derechos de importación, la devolución del Impuesto al Valor Agregado a exportadores e instrumentos de apoyo crediticio. 
más, se encuentra que la innovación de procesos (específicamente, outsourcing) y el entrenamiento de trabajadores en operaciones de exportación contribuyen a incrementar la probabilidad de exportar permanentemente. Finalmente, el desempeño inicial de los exportadores, medido en términos de valor exportado y número de mercados a los que exportan, está relacionado positivamente con el éxito exportador.

Diferenciando por el tipo de instrumento, se encuentra que la participación en comités exportadores aumenta la probabilidad de exportar persistentemente, no así los otros instrumentos. Esto sugiere implicaciones interesantes para la política de promoción de exportaciones. Primero, los esfuerzos conjuntos entre empresas pueden lograr resultados más duraderos, lo que potencialmente estaría relacionado a que los Comités facilitan la internalización de costos al reducir la posibilidad de comportamiento estratégico (free-riding). Segundo, las intervenciones públicas de carácter más sistemático generan mayores resultados que esfuerzos aislados, ya que facilitan al aprendizaje entre empresas y con la agencia encargada. No obstante lo anterior, y un aspecto que no se analiza en Álvarez (2004), es que puede existir un problema de auto-selección: las empresas mejores y más motivadas se orientan hacia la formación de comités y/o la agencia encargada selecciona las empresas mejores.

Un reciente e importante trabajo en esta literatura es el realizado por Volpe Martincus y Caballo (2010), que utilizan información para todos los exportadores Chilenos, incluyendo exportaciones totales desglosadas por producto y país de desti$\mathrm{no}^{10}$. Esto es complementado con información de si las empresas han participado en las actividades de promoción organizadas por PROCHILE durante el período 20022006. Este trabajo realiza tres contribuciones importantes respecto a los trabajos previos. Primero, utiliza información para el universo de empresas exportadoras y no está sujeto a los problemas de muestra reducida que caracterizan a los estudios analizados anteriormente. Segundo, utilizan una metodología de evaluación de impacto con datos de panel y aplican técnicas de propensity score matching junto con un análisis de diferencias en diferencias. Esto último reduce los sesgos asociados a factores no observados, que no varían en el tiempo, que pueden afectar la decisión de utilizar los instrumentos. Finalmente, el trabajo estima el impacto en diferentes tramos de la distribución de tamaño de las empresas, lo que entrega evidencia respecto a las potenciales heterogeneidades en el efecto de estos instrumentos.

Sus resultados básicos indican un efecto promedio positivo de la participación en PROCHILE en cuatro de los seis indicadores de desempeño exportador evaluado. Se encuentra que la utilización de estos instrumentos favorece el crecimiento de las exportaciones y el crecimiento del número de países de destino, así también como el crecimiento de las exportaciones por mercado y por producto. No se encuentra un efecto significativo sobre el crecimiento de las exportaciones por producto y mercado, y tampoco sobre el crecimiento en el número de productos. Luego, aplicando

10 Otro trabajo reciente es el de AGOSIN et al. (2009), que evalúa los efectos de un instrumento de promoción de exportaciones denominado reintegro simplificado. 
técnicas semi-paramétricas para estimar el efecto por deciles de la distribución de las variables de resultados, estos autores encuentran que las actividades de promoción realizadas por PROCHILE han tenido efectos diferenciados. Los efectos positivos tienden a estar concentrados principalmente en las empresas con crecimiento más bajos de las exportaciones totales y en aquellas con crecimientos altos y bajos en el número de países de destino y número de productos. Indagando en las características de estas empresas relativamente más beneficiadas por la utilización de los instrumentos de promoción, Volpe Martincus y Caballo (2010) encuentran que éstas corresponden a las empresas más pequeñas (medidas por sus exportaciones totales). Los autores concluyen que esta evidencia es consistente con la idea que las barreras asociadas a la internacionalización son mayores en las empresas más pequeñas, y es justamente en ellas donde los programas de promoción tienen un efecto más relevante.

En resumen, la evidencia para Chile, usando distintas fuentes de información y metodologías, indica que los instrumentos de promoción de exportaciones han tendido a favorecer el desempeño exportador de las firmas. No obstante, los efectos tienden a ser heterogéneos por tipo de instrumento, por indicador de desempeño evaluado y por características de las empresas.

\section{Conclusiones e implicaciones de política}

Este trabajo ha tenido como objetivo extraer algunas lecciones sobre los efectos de la promoción de exportaciones en el desempeño de las firmas chilenas, utilizando varios estudios realizados en la última década. En primer lugar, se revisó el fundamento de la política de promoción de exportaciones basado en la idea de fallas de mercado. En teoría, problemas de información y externalidades tecnológicas han sido el fundamento de estas políticas. No obstante, es difícil encontrar modelos teóricos formales que explícitamente consideren este tipo de fallas de mercado y sirvan como base para el análisis empírico y la discusión de las políticas más apropiadas. Hasta ahora, el análisis empírico, aunque recientemente mejorado por la aplicación de técnicas modernas de evaluación de impacto, tiende a ser bastante ad-hoc y no rigurosamente fundamentado por un modelo teórico que permita extraer implicaciones a ser testeadas empíricamente.

Relacionado a lo anterior, la evidencia empírica sobre estas fallas de mercado no es completamente concluyente. Aun cuando existen varios estudios analizando la existencia de externalidades de la intensidad exportadora de firmas domesticas y extranjeras sobre el desempeño de firmas en la misma industria e industrias relacionadas, no existe un consenso respecto a la relevancia de las externalidades originadas por la actividad exportadora. Como ha sido sugerido por Greenaway y Kneller (2007), en su revisión de varios de estos trabajos, esto puede deberse a diferencias metodológicas en la forma en que se miden las variables relacionadas a las externalidades, pero también persiste el desafío no menor de identificar causalmente este 
tipo de relaciones. La mayoría de estos trabajos no ha sido capaz de convincentemente mostrar que una relación positiva entre, por ejemplo, la probabilidad de exportar de una firma y/o su productividad, y la presencia de exportadores en la industria se debe a la existencia de externalidades, o puede ser el reflejo de shocks comunes no controlados o sencillamente a un fenómeno de autoselección. En este sentido, existen desafíos importantes en esta área de investigación para tratar de identificar la existencia de estas externalidades y tener un mejor fundamento para la adopción de políticas tendientes a solucionar estas fallas de mercado potenciales.

En general, los distintos trabajos realizados sobre la experiencia chilena en promoción de exportaciones, principalmente basados en la utilización de los instrumentos de PROCHILE, indica que la promoción de exportaciones ha tendido a favorecer el desempeño exportador de las firmas. Como queda claro de la revisión de la literatura, este resultado es relativamente robusto al uso de distintas fuentes de información y metodologías. Incluso, la utilización de técnicas modernas de evaluación de impacto revela efectos positivos de estos instrumentos. No obstante, esta literatura sugiere que los efectos tienden a ser heterogéneos por tipo de instrumento, por indicador de desempeño evaluado y por características de las empresas. Esto puede tener implicancias importantes para la implementación de este tipo de políticas.

En primer lugar, sugiere que algunos instrumentos son más efectivos que otros. Los primeros trabajos realizados en Chile indican que la participación en comités exportadores fue la que efectivamente contribuyó a mejorar el desempeño exportador de las firmas. Ello sería consistente con la idea que los esfuerzos conjuntos entre empresas y las intervenciones públicas de carácter más sistemático son las que generan mejores resultados. No obstante, tampoco puede descartarse que esto se deba a un fenómeno de selección de las mejores y más motivadas empresas. En segundo lugar, esta evidencia señala que no es trivial el tipo de indicador que se utiliza en la evaluación, con lo cual sería necesario contar con indicadores complementarios que contribuyan a tener una mejor visión de los efectos de los programas.

Finalmente, este tipo de evaluaciones, aunque útiles para identificar la efectividad de estos instrumentos, no están diseñadas para responder explícitamente a preguntas respecto a la rentabilidad social de los fondos públicos asignados. Aun cuando se pueden realizar algunas aproximaciones usando información del gasto realizado por las agencias de promoción y las propias empresas, y comparar éstos con los beneficios medibles en las empresas participantes, no es suficiente para tener una medida más precisa del beneficio para el país de la asignación de recursos públicos a este tipo de actividades. Avanzar en este sentido puede ser importante para la priorización del gasto público en sus distintos usos alternativos. 


\section{Referencias bibliográficas}

[1] AGOSIN, M.R.; LARRAÍN, CH. y GRAU, N. (2009): «Industrial Policy in Chile», Documento de Trabajo 294, Departamento de Economía, Universidad de Chile.

[2] AITKEN, B.; HANSON, G. H. y HARRISON, A. E. (1997): «Spillovers, Foreign Investment and Export Behavior», Journal of International Economics 43 (1-2): 103132.

[3] ÁLVAREZ, R. (2004): «Sources of Export Success in Small and Medium-Sized Enterprises: The Impact of Public Programs», International Business Review, 13, 2004, pp. 383-400.

[4] ÁLVAREZ, R. y LÓPEZ, R. A. (2008): «Is Exporting a Source of Productivity Spillovers», Review of World Economics, 144(4), 723-749

[5] ÁLVAREZ, R. y CRESPI, G. (2000a): «Efecto de las Políticas de Fomento en el Dinamismo Exportador Chileno», El Trimestre Económico, 268 (4): 557-577.

[6] ÁlVAREZ, R. y CRESPI, G. (2000b): «Exporter Performance and Promotion Instruments: The Chilean Empirical Evidence», Estudios de Economia, 27 (2): 225-241.

[7] ARSLAN, I. y WIJNBERGEN,S. (1993): «Export Incentives, Exchange Rate Policy and Export Growth in Turkey», The Review of Economics and Statistics, 75 (1): 1281333.

[8] BARRIOS, S.; GÖRG, H. y STROBL, E. (2003): «Explaining Firms' Export Behavior: R\&D, Spillovers andthe Destination Market», Oxford Bulletin of Economics and Statistics 65 (4): 475-496.

[9] BERNARD, A. B. y JENSEN, J. B. (2004): «Why Some Firms Export», Review of Economics and Statistics 86 (2): 561-569.

[10] BLYTHE (1996): «The Evolution of Non Selling Activities at British Trade Exhibitions», Marketing Intelligence and Planning, 14 (5): 20-14.

[11] CAVUSGIL, T. y NAOR J. (1987): «Firm and Management Characteristics as Discriminators of Export Marketing Activity», Journal of Business Research, 15 (3): 22123

[12] CLERIDES, S. K.; Lachy, S. y TYBOUT, J. R. (1998): «Is Learning by Exporting Important? Micro-Dynamic Evidence from Colombia, Mexico and Morocco», Quarterly Journal of Economics 113(3): 903-947.

[13] COUGHLIN, C. y CARTWRIGHT, P. (1987): «An Examination of State Foreign Export Promotion and Manufacturing Exports», Journal of Regional Science, 27(3): 439-449.

[14] EDWARDS, S. (1993): «Openness, Trade Liberalization, and Growth in Developing Countries», Journal of Economic Literature, 31 (3): 1358-1393.

[15] FEDER, G. (1982): «On Exports and Economic Growth», Journal of Development Economics, 12 (1-2):59-73.

[16] GIRMA, S.; Görg, H. y Pisu, M. (2008): «Exporting, Linkages and Productivity Spillovers from Foreign Direct Investment», Canadian Journal of Economics, 41 (1): 320-340.

[17] GÖRG, H. y HIJZEN, A. (2004): «Multinationals and Productivity Spillovers», GEP Research Paper 04/41, University of Nottingham.

[18] GREENAWAY, D. y Kneller, R. (2008): «Exporting, Productivity and Agglomeration», European Economic Review, 52(5): 919-939. 
[19] GREENAWAY, D.y KNELLER, R. (2007): «Firm Heterogeneity, Exporting and Foreign Direct Investment», Economic Journal, 117 (517): 134-161.

[20] GREENAWAY, D.; SOUSA, N. y WAKELIN, K. (2004). Do Domestic Firms Learn to Export fromMultinationals?», European Journal of Political Economy 20 (4): 10271043.

[21] HAUSMANN, R. y Rodrik, D. (2003): «Economic Development as Self-Discovery», Journal ofDevelopment Economics, 72 (2): 603-633.

[22] JAVORCIK, B. S. (2004): «Does Foreign Investment Increase the Productivity of Domestic Firms? InSearch of Spillovers through Backward Linkages», American Economic Review, 94 (3): 605-627.

[23] KARPATY, P. y KNELLER, R. (2005): «Demonstration or Congestion? Export Spillovers in Sweden», GEP Research Paper 05/44, University of Nottingham.

[24] KEESING, D. B. (1967): «Outward-Looking Policies and Economic Development», Economic Journal, 77 (306): 303-320.

[25] KNELLER, R. y PISU, M. (2007): «Industrial Linkages and Export Spillovers from FDI», The World Economy, 30 (1): 105-134.

[26] LALL, S. (2002): «Selective Policies for Export Promotion: Lessons from the Asian Tigers», en G. K.Helleiner (ed.), Non-Traditional Exports and Development in SubSaharan Africa: Experience and Issues. Londres: Palgrave.

[27] LEDERMAN, D.; OLARREAGA, M. y PAYTON, L. (2010): «Export Promotion Agencies: Do They Work», Journal Development of Economics, 91 (2): 257-265.

[28] LOW, P. (1982): «Export Subsidies and Trade Policy: The Experience of Kenya», World Development, 10 (4): 293-304.

[29] MARKUSEN, J. R. y VENABLES, A. J. (1999): «Foreign Direct Investment as a Catalyst for IndustrialDevelopment», Journal of International Economics, 43 (2): 335 356.

[30] MARKUSEN, J. R.y TROFIMENKO, N. (2007): «Teaching Locals New Tricks: Foreign Experts as Channel of Knowledge Transfers», NBER Working Paper 12872. National Bureau of Economic Research.Cambridge, MA.

[31] PANAGARIYA, A. (2000): «Evaluating the Case for Export Subsidies», Policy Research Working Paper 2276, World Bank. Washington, DC.

[32] PFEIFFER, R.; BURGEMEISTER, H.; HIBBERT, E. y SPENCE, M. (1998): «A Comparative Survey of Trade Fairs in the U.K. and Germany in Three Industry Sectors», Journal of European Business Education, 7 (2): 52-69.

[33] RODRIGUEZ-CLARE, A. (1996): «Multinationals, Linkages, and Economic Development», American Economic Review, 86 (4): 852-873.

[34] RODRIK, D. (1999): «New Global Economics and Developing Countries: Making Openness Work», Policy Essay No. 24. Washington, D.C.: Overseas Development Council. Baltimore: JohnsHopkins University Press.

[35] RUANE, F. y SUTHERLAND, J. (2005): «Foreign Direct Investment and Export Spillovers: How DoExport Platforms Fare?», IIIS Discussion Paper No. 58, Institute for International IntegrationStudies (IIIS), Trinity College, Dublin.

[36] SHIPLEY, C.; EGAN, C. y WONG, K. (1993): «Dimension of Trade Show Exhibiting Management», Journal of Marketing Management, 9: 55-63.

[37] SJÖHOLMM, F. (2003): «Which Indonesian Firms Export? The Importance of Foreign Networks», Papers in Regional Science, 82 (3): 303-425 
[38] SPENCE, M. (2003): «Evaluating Export Promotion Programmes: U.K. Overseas Trade Missions and Export Performance», Small Business Economics, 20 (1): 83-103.

[39] VOLPE MARTINCUS, C. (2010): «Odyssey in International Markets : An Assessment of the Effectiveness of Export Promotion Activities in Latin America and the Caribbean», Banco Interamericano del Desarrollo, Washignton D.C.

[40] VOLPE MARTINCUS, C. y CARBALLO, J. (2010): «Beyond the Average Effects: The Distributional Impacts of Export Promotion Programs in Developing Countries», Journal of Development Economics, 92 (2): 201-214.

[41] WESTPHAL, L. E. (1990): «Industrial Policy in an Export Propelled Economy: Lessons from South Korea's Experience», Journal of Economic Perspectives, 4 (3): 41 59.

[42] WILKINSON, T. y BROUGHTERS, L. E. (2006): «Trade Promotion and SME Export Performance», International Business Review, 15 (3): 233-252.

[43] WILKINSON, T. y BROUTHERS, L. E. (2000): «An Evaluation of State Sponsored Promotion Programs», Journal of Business Research, 47 (3): 229-236. 